\title{
ENDOSCOPIC CARTILAGE TYMPANOPLASTY. OUR TECHNIQUE AND EXPERIENCE OF 157 CASES
}

\author{
Muhammad Atif Najam, Urwa Sarwar, Maqbool Raza*, Khalid Azam Khan**, Humaira Saleem, Najm u Saqib Niazi, \\ Muhammad Rashid, Naeem Riaz ${ }^{* * *}$ \\ Combined Military Hospital Kharian/National University of Medical Sciences (NUMS) Pakistan, *Combined Military Hospital Multan/National University of \\ Medical Sciences (NUMS) Pakistan, ${ }^{* *}$ Combined Military Hospital Lahore/National University of Medical Sciences (NUMS) Pakistan, \\ ***Pakistan Naval Ship Hafeez, Islamabad Pakistan
}

\begin{abstract}
Objective: To assess the graft take rate and hearing gain in trans-canal endoscopic tympanoplasty Study Design: Descriptive study.

Place and Duration of Study: Pakistan Naval Ship Hafeez, Islamabad Pakistan, form Dec 2015 to Jun 2018

Methodology: All patients with dry central perforations of $>3$ months history were included in the study. All Patients with perforations of pars tensa were booked for endoscopic cartilage tympanoplasty. All perforations were divided into four types small, medium, large and subtotal or total based on size of the tympanic membrane perforations. Small perforations involving $<25 \%$ area of Pars Tensa. Medium perforation are Perforations involving 25-50\% of Tympanic membrane. Large perforations include Perforations involving $50-75 \%$ of pars tensa. Subtotal or total perforations are defined as perforations with $>75 \%$ perforation of the tympanic membranes. Wet Ears and perforations involving the Pars Flacida were excluded from the study. Preoperative Audiogram was carried out for all patients and A-B Gap were noted for all groups separately so as the demographic data including age and gender. Success was defined as complete closure of Tympanic membrane perforation at 3 months. All patients were operated with 0 Degree $3 \mathrm{~mm}, 14 \mathrm{~cm}$ rigid endoscope under general anesthesia.

Results: Mean age of patients was. 37 years and standard deviation was 12.34 (range 12-58 years). Out of total 157 cases 81 patients male were males (51\%) and 76 patients were females (49). Out of 157 ears operated 146 Perforations healed completely an overall success rate of $92 \%$. Patients with small perforations had 100 percent success rate whereas near total or total perforations had $86 \%$ Success rate. Average preoperative A-B gap was $35 \mathrm{db}$ and Average Postoperative A-B Gap was 15db. Ossicular discontinuity was noted in $34(21.6 \%)$ cases and occlusion of ventilation including obstruction of eusthachian-tube opening was noted in $61(38 \%)$ cases. Average Operative was 67 minutes. Incision scar was visible only in 2 patients 3 months after surgery.

Conclusion: Endoscopic trans canal cartilage tympanoplasty is scarless safe effective procedure with demonstrable tympanic membrane perforation closure rates and hearing gains in all patients with minimum complications. The Technique is single handed and has steep learning curve.
\end{abstract}

Keywords: Endoscopic ear surgery, Hearing loss, Pars tensa, Tragal cartilage, Tympanic membrane perforation.

How to Cite This Article Najam MA, Sarwar U, Raza M, Khan KA, Saleem U, Niazi N, Rashid M, Riaz N. Endoscopic Cartilage Tympanoplasty. Our Technique and Experience of 157 Cases. Pak Armed Forces Med J 2021; 71 (Suppl-3): S440-444. $\quad$ Doi: https://doi.org/10.51253/pafmj.v1i1.7935

This is an Open Access article distributed under the terms of the Creative Commons Attribution License (https://creativecommons.org/licenses/by-nc/4.0/), which permits unrestricted use, distribution, and reproduction in any medium, provided the original work is properly cited.

\section{INTRODUCTION}

Tympanoplasty is defined as removing the disease from the middle ear and reconstructing the tympanic membrane with or without ossiculoplasty. ${ }^{1}$ Microscopes are being used for tympanoplasty since the early $1900 . .^{2}$ The introduction of endoscopes in otological practice has revolutionized middle ear surgery by virtue of not only better visualization but also being able to surgically improve middle ear ventilation pathways that are usually cause of chronic middle ear infections leading to Tympanic membrane perforations. ${ }^{3}$ Wullstein divided tympanoplasty into five, ${ }^{2}$ types where type I is repair of tympanic membrane perforation only. Type II includesossicular discontinuity tympanic

Correspondence: Dr Muhammad Atif Najam, ENT Department, Combined Military Hospital Kharian Pakistan membrane grafted over the incus. InType IIIGraft is placed directly over the stapes head. Type IV tympanoplasty includes placing the graft over foot plate of stapes. Type $\mathrm{V}$ includes repair of tympanic membrane with fixed foot plate of stapes. Various Grafts have been used for repairing the tympanic membrane of which temporalis fascia and tragal cartilage, ${ }^{4}$ arethe most popular due to easy availability at the site of surgery along with low metabolic rate. ${ }^{5}$ Two techniques have popularly been used in tympanoplasty namely on lay and underlay with equal success rates. ${ }^{6}$ Traditionally microscopes have been used for tympanoplasty and because of limited field of view and bony canal variations more invasive post aural exposures were used to repair anterior perforations. ${ }^{7}$ Muaz et al created a working group of endoscopic ear surgery 
1999 started using endoscopes as a single tool for treating middle ear diseases. ${ }^{8}$ Endoscopes have the advantage of wider field of view, superior exposure, evaluation and management of complex middle ear anatomy. Endoscopes have an added advantage of addressing the ventilation pathways during tympanoplasty which are difficult to visualize assess and treat with a limited field of view offered by microscopes. ${ }^{3}$ We share our experience of 157 endoscopic cartilage tympanoplasty by far largest series on the subject from Pakistan. We present our technique, success rate, hearing results and ossicular and ventilation abnormalities encountered during surgery. Tragal cartilage was preferred graft because of its availability in adequate quantity from the operative field, natural concavity for reconstructing the tympanic membrane and incision on medial unexposed site of tragus.

\section{METHODOLOGY}

This Descriptive study was carried out at Pakistan Naval Ship Hafeez Islamabad, from December 2015 to June 2018. A total of 157 cases of endoscopic cartilage tympanoplasty were included in the study.

Inclusion Criteria: Patients of both genders over 12 years fit for general anaesthesia were included in the study All cases with dry perforation in the pars tensa were included in the study. The patients were categorized into 4 groups based on size of perforation. Group A included patients with small sized perforation involving upto 25 percent of parstensa. Patients in group B had medium sized perforation involving $25-50 \%$ pars tensa. Group C had 50-75 perforation in the pars tensa and group D had near total perforation with only rim of pars tensa intact. Preoperative Audiogram was done for all cases and A-B Gap noted.

Exclusion Criteria: All cases with active discharge, involvement of pars flacida were excluded from the study. Endoscopic tympanoplasty was performed using a 0 Degree $3 \mathrm{~mm} 14 \mathrm{~cm}$ rigid endoscope with 3chip Camera and medical grade monitor. Tragal cartilage was harvested using a $2-3 \mathrm{~cm}$ incision on the medial side of tragus. Skin elevatedon both sides of the cartilage. Adequate sized cartilage was harvested sparing a superior rim of cartilage to prevent cosmetic deformity at the tragus. Cartilage was harvested with perichondrium on both sides. Perichondrium on the lateral side was stripped of and prepared as a separate graft. Inicion was closed with $5 / 0$ prolene sutures. $1 \%$ Lignocaine with adrenaline Local anaethesia was infilterated at $6^{\circ}$ clock and $12^{\circ}$ clock position at the junction of cartilaginous and bony canal with and insulin syringe under endoscopic vision. Margins of perforation freshened with sharp curved needle. Canal incison was made $5 \mathrm{~mm}$ from the bony annulus from $6-12^{\circ}$ clock position. Tymapnomeatal flap was elevated with help of rosen knife. Fibrous annulus and chorda tympani identified and middle ear mucosa incised using curved needle. Tympanomeatal flap lifed of the handle of malleus and flap reflected superiorly. Four quadrant middle ear examination done with 0 and 30 degree endoscopes. Opening of eustachian tube, processuscochleaformis, anterior and posterior ventilation pathways, sinus tympani and ossicles examined. Ossicular status and mobility noted. Middle ear bed was prepeared with gelfoam and full thickness tragal cartilage cut to appropriate size with sculptured groove for handle of malleus placed via underlay technique the side of cartilage denuded of perichondrium facing laterally. Perichondrium graft placed over the cartilage. Tympanomeatal flap repositioned and external auditory canal filled with gelfoam and packed with small bismithiso examined Fat from ear lobule was harvested from a small posterior incision for sealing the stapedotomy and wound was closed with 5/0 prolene sutures. Transcanal approach was used for all cases. Canal incisions were made at $12-6^{\circ}$ clock and tympanomeatal flap was elevated chorda tympani was identified middle ear entry was made. Posterior bone removal was done with a curette or micro drill. Pyramidal process, Stapedius tendon, facial nerve stapes supra structure and foot plate was exposed. Stapedius tendon was divided with micro scissors (Figure-1). Incudo-stapedial joint was divided with joint knife. Crurotomy and stapedotomy was done with $0.7 \mathrm{~mm}$ diamond burr using a stapes drill (Figure-2). Footplate distance from the incus was measured and appropriate size Teflon piston $0.6 \mathrm{~mm}$ diameter was placed in the stapedotomy (Figure-3). Piston position and mobility was confirmed with bending sign (Figure-4) and round window reflex. Fat graft was placed around the piston to seal stapedotomy (Figure-5). Tymapnomeatal flap was repositioned and spongeston was filled in the external auditory canal. Operative time was noted from start of transcanalincision's to repositioning of tympanomeatal flap. and Postoperative facial nerve function and nystagmus was documented. Post operative follow up was done after 2, 4 and 12 weeks. Postoperative audiogram was carried out at 12 weeks along with confirmation of graft take and rejection. Ossicular and middle ear ventilation pathway abnormalities noted along with total operative time. Ossiculoplasty was done in all patients with ossicular discontinuity. Remenant incus or teflon 
prosthesis were used for ossiculoplasty. Presence of visible scar with naked eye at 3 months was also noted. All data was entered in SPSS and mean was calculated for Age, Gender, A-B Gap, operative time, success of graft uptake middle ear abnormalities and visible scar was calculated.

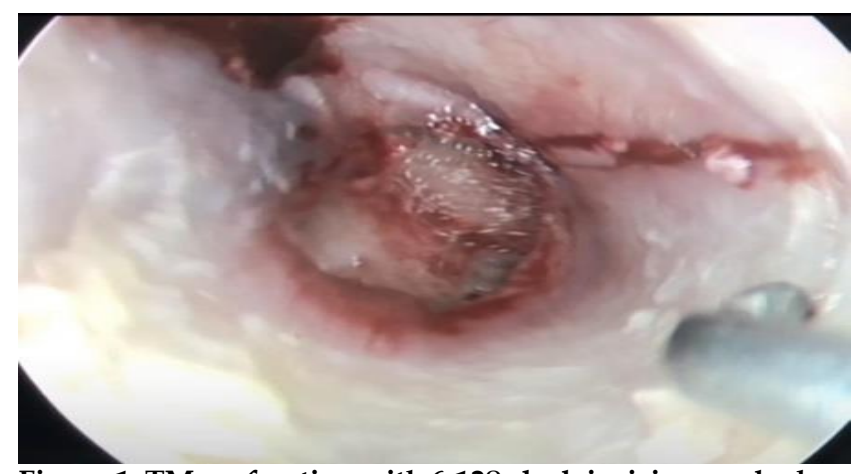

Figure-1: TM perforation with $6-12^{\circ}$ clock incision marked.

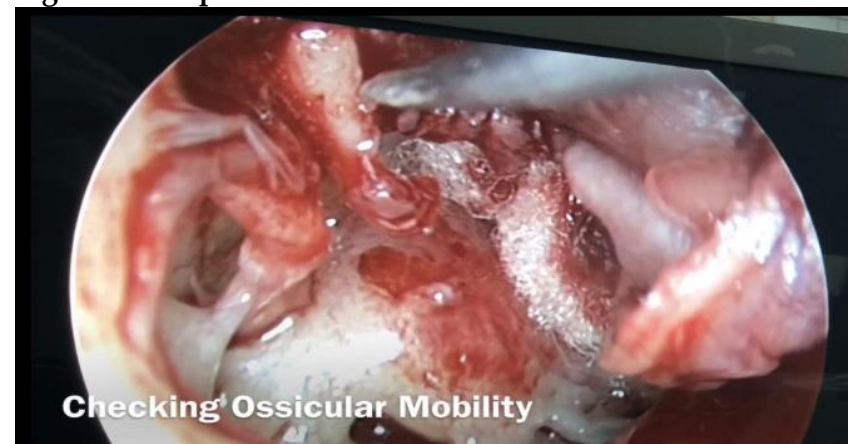

Figure-2: Malleus denuded and ossciular integrity checked.

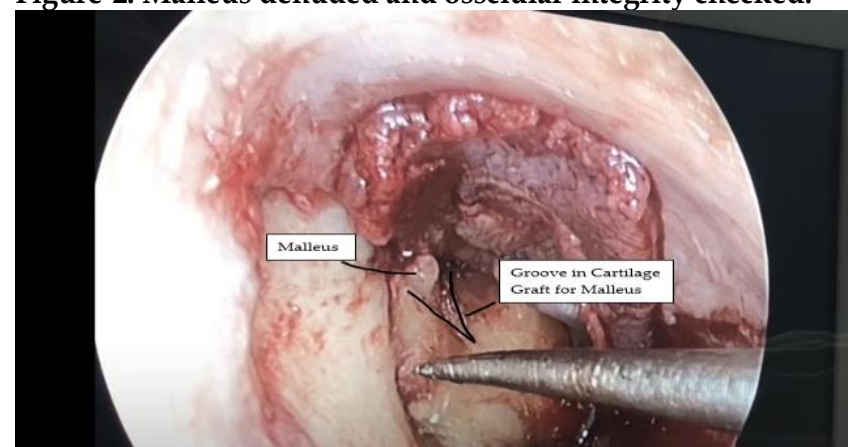

Figure-3: Cartilage graft placed with slit for malleus.

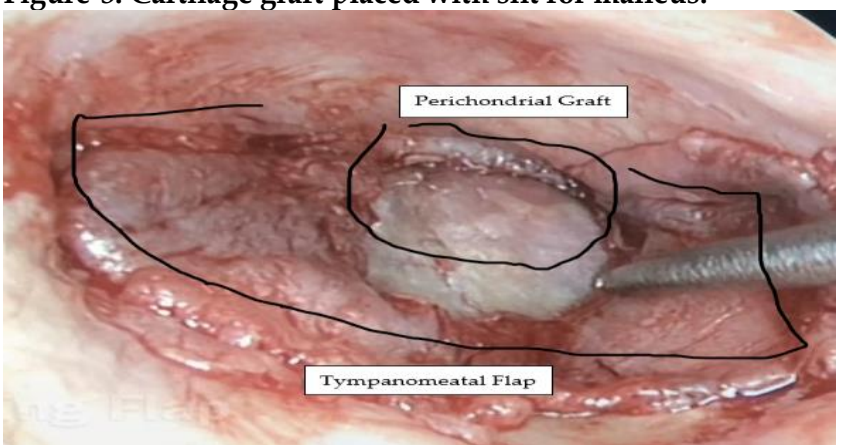

Figure-4: Perichondrium graft placed and tympanomeatal flap repositioned.

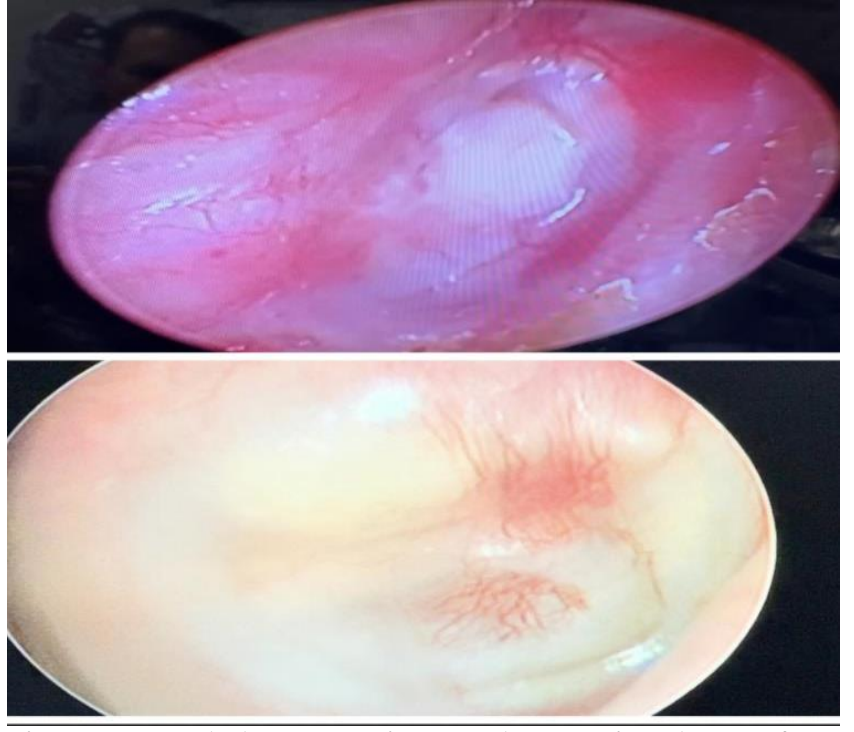

Figure-5: Healed Tympanic membranes in three of our patients.

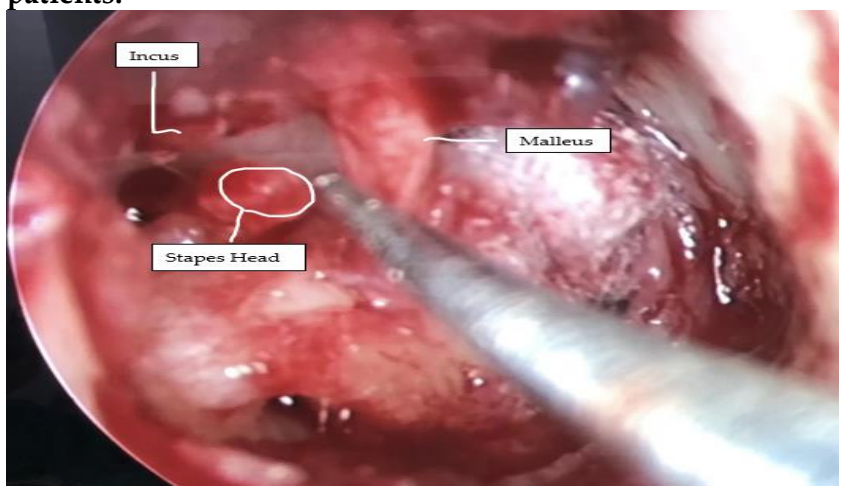

Figure-6: Intraoperative incudo-stapedial discontinuity.

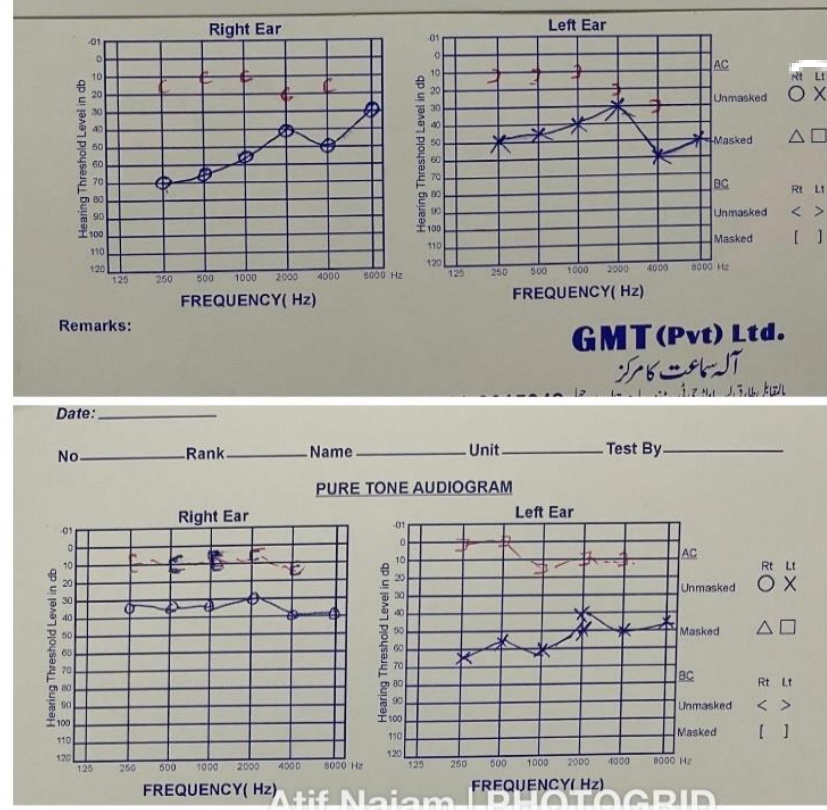

Figure-7: Preoperative and postoperative audoigram in a patient with ossicular reconstruction. 


\section{RESULTS}

Total 157 patients were included in the study Mean age of patients was 37 years and standard deviation was 12.34 (range 12-58 years). Out of 81 were males and 76 females. Among the 157 patients operated 17 (10.82\%) had small, 42 ( 26.75\%) medium, 69 (43.9\%) large and $29(18.4 \%)$ had subtotal or near total tympanic membrane perforation. Preoperative Average Air bone gap was $35 \mathrm{db}$. Tympanic membrane closure rate was $17(100 \%)$ for small, 40 (95\%)for medium, 64 (92\%) for large and $25(86 \%)$ for near total and total perforations. Over all 146 (93\%)patients had complete tympanic membrane closure seen in our series. Out of 39 $(24.8 \%)$ patients had ossicular discontinuity out which $23(14.64 \%)$ patients had necrosis of long process of incus, $10(6.3 \%)$ patients had necrosis of supra-structure of stapes and $6(3.8 \%)$ patients had necrosis of handle of malleus. Four $(2.57 \%)$ patients had sclerosis involving the stapes suprastructure, incus and foot plate. A total of $49(31 \%)$ patients had blockage in the ventilation pathways. The Ventilation pathway pathologies included granulations in the anterior and posterior isthmuses and fibrous bands obstructing the eustachian tube ope-ning.Postoperativeaverage A-B gap was $13 \mathrm{db}$. Of $72 \%$ patients had $>20 \mathrm{db}$ of A-B Gap reduction, $18 \%$ patients had $12-15 \mathrm{db}$ of A-B Gap closure where as $10 \%$ patients had $<10 \mathrm{db}$ of A-B Gap reduc-

Table: Perforation closure and hearing gain.

\begin{tabular}{l|c|c|c|c|c|c}
\hline $\begin{array}{l}\text { Size of } \\
\text { Perforation }\end{array}$ & $\begin{array}{c}\text { Total Cases } \\
\mathbf{1 5 7}\end{array}$ & $\begin{array}{c}\text { Males } \\
\mathbf{8 1}\end{array}$ & $\begin{array}{c}\text { Females } \\
\mathbf{7 6}\end{array}$ & $\begin{array}{c}\text { Complete } \\
\text { Closure }\end{array}$ & $\begin{array}{c}\text { Average Pre-perative } \\
\text { A-B Gap }\end{array}$ & $\begin{array}{c}\text { Average Post Operative } \\
\text { A-B Gap }\end{array}$ \\
\hline Small & 17 & 11 & 6 & $17(100 \%)$ & $10 \mathrm{db}$ & $08 \mathrm{db}$ \\
\hline Medium & 42 & 22 & 20 & $40(95 \%)$ & $15 \mathrm{db}$ & $10 \mathrm{db}$ \\
\hline Large & 69 & 35 & 34 & $64(92 \%)$ & $25 \mathrm{db}$ & $10 \mathrm{db}$ \\
\hline Total/Near Total & 29 & 13 & 16 & $25(86 \%)$ & $30 \mathrm{db}$ & $15 \mathrm{db}$ \\
\hline
\end{tabular}

tion. Mean operative time was 62 minutes.

\section{DISCUSSION}

Traditionally tympanoplasties have been performed with microscopes. ${ }^{2}$ Endoscopes is the lattest tool for surgical management of middle ear pathologies. Endoscopic tympanoplasty has rapidly gained popularity among ENT surgeons because of better visualizations of middle ear anatomy, completely trans-canal approach, no need for canaloplasty in most cases and avoidance of endaural and post-aural incisions. Tragal cartilage is a robust graft for middle ear reconstruction. Initially was reserved for total perforations and revision surgeries but currently its being used for primary tympanoplasties for its better take rates and hearing gains. ${ }^{9}$ Tragal cartilage is a favored graft for endosco- pic ear surgeons for the reason its easy to handle with a single hand technique and readily available in operative site along with better cosmetic result. Success rate of endoscopic tympanoplasty has been reported between $90-98 \%$ depending on the size and site of perforations. ${ }^{10}$ Our study has reported an overall success rate of $92 \%$ with $100 \%$ success rate for small perforations and $86 \%$ for total perforations. Our results are similar to quoted by choi et al,11 and Nausheen et al, in their studies. ${ }^{12}$ The mean operative time for endoscopic ear surgery has been reported between 50-65 minutes. ${ }^{13-14}$ Operative time in our study was 62 minutes. Tragal cartilage is associated with good hearing results. Many studies have quoted significant hearing gains with full thickness tragal cartilage owing to its natural curvature for use in middle ear and average $5 \mathrm{~mm}$ thickness. ${ }^{15}$ Our study also has shown $90 \%$ patients having more than $12 \mathrm{db}$ A-B gap closure suggesting a perceptible hearing gain. Vershney et al ${ }^{16}$ reported $7.7 \%$ incidence of ossicular injury in mucosal type of chronic suppurative otitis media. In our study $24.8 \%$ patients had ossicular discontinuity of which commonest was necrosis of long process of incus similar to reported by vershney et al. ${ }^{16}$ Middle ear ventilation has been decribed in detail by tarabichi and marchioni, ${ }^{17-18}$ and addressing the ventilation pathways is mandatory for a successful middle ear surgery. In $31 \%$ of our patients we noticed an obstruction in the ventilation pathways of the middle ear cleft in form of polyp, granulation tissue or inflamed hypertrophic middle ear mucosa. It's now considered paramount to address these ventilation pathways for a successful tympanoplasty. These ventilation pathways were poorly visualized because of operating microscopes but endoscopic ear surgery has made it possible to not only treat the structural defects of middle ear but also address physiologic disorders owing to better field of view excellent anatomic details.

\section{LIMITATION OF STUDY}

The study is first local publication on endoscopic tympanoplasty and has no local references for comparison.

\section{CONCLUSION}

Endoscopic tympanoplasty is a minimally invasive procedure that provides excellent graft take rates and comparable hearing results with additional benefit of short operative 


\section{Endoscopic Cartilage Tympanoplasty}

time minimal scar. Endoscopic ear surgery has evolved as future of otological surgical practice.

\section{Conflict of Interest: None.}

\section{Authors' Contribution}

MAN: Operating surgeon, data collection, US: Data collection, data entry, discussion writing, MR: Data collection, KAK: Data collection and results, US: Data collection, data entry, discussion writing, NN: Results complilation, MR: Data collection, data entry and discussion writing, NR: Discussion and results compilation.

\section{REFERENCES}

1. Sarkar S. A review on the history of tympanoplasty.Ind J Otolaryngol Head Neck Surg 2013; 65(Suppl-3): 455-460.

2. Wullstein $\mathrm{H}$. The restoration of the function of the middle ear, in chronic otitis media. Ann Otol Rhinol Laryngol 1956; 65(4): 10211041.

3. Pollak N. Endoscopic and minimally-invasive ear surgery: A path to better outcomes. World J Otorhinolaryngol Head Neck Surg 2017; 3(3): 129-135.

4. Yamamoto E, Iwanaga M, Fukumoto M. Otolaryngol Head Neck Surg 1988; 98(6): 546-551.

5. Hamed M, Samir M, El Bigermy M, Auris Nasus Larynx 1999; 26(3): 257-262.

6. SHEA JJ. A comparison of endoscopic and microscopic techniques for the repair of tympanic membrane perforations. Laryngol Otol 1960; 74(1): 358-362.

7. Ahmed LA, Raza SS. Tympanoplasty type-1. Endaural or postaural approach. Should the patient decide? Saudi Med J 2006; 27(12): 1847-1849.
8. Tarabichi M. Endoscopic middle ear surgery. Ann Otol Rhinol Laryngol 1999; 108(1): 39-46.

9. El-Sheikh MM. Evaluation of hearing outcome of tympanoplasty using cartilage graft versus temporalis fascia graft. Egypt J Otolaryngol 2019; 35(2):1-5.

10. Özdemir D, Özgür A, Akgül G, Çelebi M, Mehel DM, Yemiş T. Outcomes of endoscopic transcanal type 1 cartilage tympanoplasty. Eur Arch Otorhinolaryngol 2019; 276(12): 3295-3299.

11. Choi N, Noh Y, Park W. Comparison of endoscopic tympanoplasty to microscopic tympanoplasty. Clin Exp Otorhinolaryngol 2017; 10(1): 44-49.

12. Qureshi N, Baig M, Parvez M, Masood S, Afzal M. Comparison of endoscopic tympanoplasty with microscopic tympanoplasty. J Rawalpindi Med Coll 2020; 24(4): 400-405.

13. Kuo CH. Comparison of endoscopic and microscopic tympanoplasty. Eur Arch Otorhinolaryngol 2017; 274(7): 2727-2732.

14. Lade H, Choudhary SR, Vashishth A. Endoscopic vs microscopic myringoplasty: a different perspective.Eur Arch Otorhinolaryngol 2014; 271(7): 1897-1902.

15. Kaya I, Turhal G, Ozturk A, Gode S, Bilgen C, Kirazli T. Results of endoscopic cartilage tympanoplasty procedure with limited tympanomeatal flap incision. Acta Otolaryngol 2017; 137(11): 1174-1177.

16. Varshney S, Nangia A, Bist SS, Singh RK, Gupta N, Bhagat S. Ossicular chain status in chronic suppurative otitis media in adults.Ind J Otolaryngol Head Neck Surg 2010; 62(4): 421-426.

17. Marchioni D, Rubini A, Soloperto D. Endoscopic ear surgery: redefining middle ear anatomy and physiology. Otolaryngo 1Clin North Am 2021; 54(1): 25-43.

18. Tarabichi M, Ayache S, Nogueira JF, Al Qahtani M, Pothier DD. Endoscopic management of chronic otitis media and tympanoplasty. Otolaryngol Clin North Am 2013; 46(2): 155-163. 